\title{
Influence of heat-loss on compressibility-driven flames propagating from the closed end of a long narrow duct
}

\author{
Vadim N. Kurdyumov ${ }^{\mathrm{a}}$, Moshe Matalon ${ }^{\mathrm{b}}$ \\ ${ }^{a}$ Department of Energy, CIEMAT, Madrid, Spain \\ ${ }^{b}$ Department of Mechanical Science and Engineering, University of Illinois, Urbana-Champaign, U.S.A.
}

\begin{abstract}
This study focuses on the dynamics of compressibility-driven flames that emerge in narrow tubes, closed at their ignition end, when conductive heat losses through the walls are appreciable. A narrow gap approximation is used to reduce the governing equations to an effectively one-dimensional problem. In long channels this problem admits traveling-wave solutions which we have investigated numerically for finite values of the Zel'dovich number, and asymptotically in the limit of large Zel'dovich numbers. In particular, we describe the flame structure and the dependence of the propagation speed on the physico-chemical parameters, including the heat loss and compressibility parameters, and examine the transition from compressibility-driven to isobaric flames when systematically reducing the representative Mach number.
\end{abstract}

Key words: Compressibility-driven flames, Isobaric flames, Heat Loss, Flames in long ducts, Activation Energy Asymptotics

\section{Introduction}

The propagation of a flame into a quiescent combustible mixture is a fundamental problem in combustion theory. The one-dimensional problem admits a traveling wave solution describing the propagation of a planar flame that separates burned products from the fresh combustible mixture and travels at a relatively low speed, generally known as the laminar flame speed. The flame structure consists of a preheat zone, where the fresh mixture is heated by conduction and the reactants drawn by diffusion, and a thin reaction zone where most of the chemical activity takes place. In the absence of heat losses, the temperature attains the isobaric adiabatic flame temperature. The representative Mach number, corresponding to the ratio of the flame speed to the speed of sound is extremely small throughout the wave, such that compressibility effects are 
negligibly small. The gas density decreases as a result of thermal expansion and the propagation occurs under practically isobaric conditions.

It was recently discovered that when compressibility effects are small, but not negligible, the one-dimensional problem admits another type of traveling wave solution that propagates at a constant speed, but much higher speed than the laminar flame speed $[1,2]$. This occurs in long tubes closed at their ignition end where, in the absence of heat losses, the temperature of the burned gas trapped behind the flame attains the adiabatic isochoric flame temperature, which is much larger than the adiabatic isobaric flame temperature. The fresh unburned gas is first compressed and heated, before it expands as a result of the heat conducted from the reaction zone, and the pressure rises significantly throughout the entire wave. The transition from compressibility-driven to isobaric flames as the Mach number is reduced, was not clearly understood in these earlier studies.

Although flame acceleration due to compressibility effects are also observed when a flame propagates from an open end tube, traveling wave solutions with constant propagation speed are not possible. When the flame propagates from an open end tube, the pressure behind the flame is not a constant because it must equilibrate with the atmospheric pressure when the burned products flow out of the tube [3]. Since the pressure, and hence the temperature of the burned gas vary spatially, a traveling wave solution is not possible. This differs from the case of isobaric flames, because the pressure in a one-dimensional setting is excluded from the problem formulation and flame propagation from an open end tube at a constant speed is possible.

In this paper, we examine the structure and dynamics of compressibility-driven flames when heat losses through the walls are appreciable. The problem is investigated numerically for finite values of the Zel'dovich number and asymptotically for large Zel'dovich numbers. The results of the asymptotic approximation were shown to agree well with the numerical results. Moreover, the asymptotic approximation was able to capture and describe the transition from fast compressibility-driven to slow isobaric flames, when systematically reducing the compressibility parameter $\Lambda$ to small enough values, a limit that was found difficult to describe numerically.

\section{General formulation}

A combustible mixture at temperature $T_{u}$ is contained in a long narrow duct of circular crosssection of radius $R$ and length $L$. At time $t=0$, the mixture is ignited at the left end of the tube 
and, as a result, a flame propagates along its axis towards the right end. The left end of the tube remains closed during the propagation while the right end is kept open with the gas exposed to atmospheric pressure. For lean mixtures, the amount of oxidizer consumed during combustion is relatively small, such that for a chemical reaction modeled by an irreversible global step,

$$
\text { Fuel }+ \text { Oxidizer } \rightarrow \text { Products }
$$

the fuel consumption rate may be expressed as $\hat{\omega}=\mathcal{B} \hat{\rho}^{n} Y \exp (E / \mathcal{R} \hat{T})$ where $\hat{\rho}$ is the density of the mixture, $Y$ is the fuel mass fraction, $\hat{T}$ is the temperature, $E$ is the overall activation energy of the chemical reaction, $\mathcal{R}$ is the gas constant, and $\mathcal{B}$ is an appropriately-defined preexponential factor. Since a one-step reaction is a gross simplification of the complex chemical reaction scheme that characterizes combustion processes, the (empirical) exponent $n$ is left arbitrary. It takes the values $n=2$, or $n=1$, when the oxidizer mass fraction $Y_{O}$, or the oxidizer concentration $\hat{\rho} Y_{O} / W_{O}$, is assumed constant (here $W_{O}$ is the oxidizer molecular weight). Often the exact value of $n$ has slight relevance, but in the present study its value appears to be important due to the role played by the density when compressibility effects become significant. We have therefore retained $n$ arbitrary, and considered below both values, $n=1,2$.

The combustion field is assumed axisymmetric, so that all variables depend on the axial distance $\hat{x}$, radial coordinate $\hat{r}$, and time $\hat{t}$. The axial and radial velocity components of the gas mixture are denoted by $\hat{u}$ and $\hat{v}$, with $\hat{\rho}, \hat{p}$ and $\hat{T}$ the density, pressure and temperature, respectively. Dimensionless variables are introduced as follows:

$$
\begin{gathered}
x=\hat{x} / \delta_{T}, \quad r=\hat{r} / R, \quad t=S_{L} \hat{t} / \delta_{T}, u=\hat{u} / S_{L}, \quad v=\hat{v} /\left(a S_{L}\right), \\
\rho=\hat{\rho} / \rho_{u}, \quad p=a^{2}\left(\hat{p}-p_{u}\right) / \rho_{u} S_{L}^{2}, \quad Y=Y / Y_{u}, \quad \theta=\left(\hat{T}-T_{u}\right) /\left(T_{a}-T_{u}\right),
\end{gathered}
$$

where $Y_{u}$ is the mass fraction of fuel with $\rho_{u}, p_{u}, T_{u}$ the density, pressure and temperature of the fresh initial mixture, $S_{L}$ is the flame speed of a planar, adiabatic and isobaric flame, $\delta_{T}=\mathcal{D}_{T} / S_{L}$ is the thermal flame thickness, $\mathcal{D}_{T}=\lambda / \rho_{u} c_{p}$ is the thermal diffusivity of the mixture with $\lambda$ the thermal conductivity and $c_{p}$ the specific heat of the mixture at constant pressure (both assumed constant), $T_{a}=T_{u}+Q Y_{u} / c_{p}$ is the adiabatic flame temperature with $Q$ the total heat of combustion, and $a=R / \delta_{T}$ is the duct radius in units of the flame thickness $\delta_{T}$. Note that the normalized temperature $\theta$ tends to zero in the fresh mixture; it is equal to one in the burned gas, under adiabatic and isobaric conditions. Finally, when the same symbol is used for both dimensional and dimensionless quantities, the one with the "hat" accent represents the dimensional value. 
Assuming constant transport properties, conservation of mass, momentum and energy in the gas phase $(0<r<1)$ take the form

$$
\begin{gathered}
\frac{\partial \rho}{\partial t}+\frac{\partial(\rho u)}{\partial x}+\frac{1}{r} \frac{\partial(r \rho v)}{\partial r}=0 \\
\rho \frac{D u}{D t}=-\frac{1}{a^{2}} \frac{\partial p}{\partial x}+\operatorname{Pr}\left\{\frac{1}{a^{2}} \frac{1}{r} \frac{\partial}{\partial r}\left(r \frac{\partial u}{\partial r}\right)+\frac{4}{3} \frac{\partial^{2} u}{\partial x^{2}}+\frac{1}{3} \frac{1}{r} \frac{\partial}{\partial r}\left(r \frac{\partial v}{\partial x}\right)\right\} \\
\rho \frac{D v}{D t}=-\frac{1}{a^{4}} \frac{\partial p}{\partial r}+\operatorname{Pr}\left\{\frac{1}{a^{2}}\left[\frac{4}{3} \frac{1}{r} \frac{\partial}{\partial r}\left(r \frac{\partial v}{\partial r}\right)+\frac{1}{3} \frac{\partial^{2} u}{\partial x \partial r}-\frac{4}{3} \frac{v}{r^{2}}\right]+\frac{\partial^{2} v}{\partial x^{2}}\right\}, \\
\rho \frac{D \theta}{D t}=\left\{\frac{\partial^{2} \theta}{\partial x^{2}}+\frac{1}{a^{2}} \frac{1}{r} \frac{\partial}{\partial r}\left(r \frac{\partial \theta}{\partial r}\right)\right\}+\frac{\gamma-1}{q} \mathcal{N}\left\{\frac{D p}{D t}+\operatorname{Pr} \Phi\right\}+\omega \\
\rho \frac{D Y}{D t}=\frac{1}{L e}\left\{\frac{\partial^{2} Y}{\partial x^{2}}+\frac{1}{a^{2}} \frac{1}{r} \frac{\partial}{\partial r}\left(r \frac{\partial Y}{\partial r}\right)\right\}-\omega \\
1+\mathcal{M} \gamma p=\rho(1+q \theta),
\end{gathered}
$$

where

$$
\frac{D}{D t} \equiv \frac{\partial}{\partial t}+u \frac{\partial}{\partial x}+v \frac{\partial}{\partial r}
$$

is the convective derivative,

$$
\Phi=\left(\frac{\partial u}{\partial r}+a^{2} \frac{\partial v}{\partial x}\right)^{2}+a^{2}\left\{2\left(\frac{\partial u}{\partial x}\right)^{2}+2\left(\frac{\partial v}{\partial r}\right)^{2}+2 \frac{v^{2}}{r^{2}}-\frac{2}{3}\left(\frac{\partial u}{\partial x}+\frac{1}{r} \frac{\partial(r v)}{\partial r}\right)^{2}\right\}
$$

is the viscous dissipation function and

$$
\omega(\rho, \theta, Y)=\frac{(1+q)^{2} \beta^{2}}{2 L e s_{L}^{2}} \rho^{n} Y \exp \left\{\frac{\beta(\theta-1)}{1+q(\theta-1) /(1+q)}\right\}
$$

is the reaction rate. The parameters appearing in these equations are: the ratio of specific heats $\gamma=c_{p} / c_{v}$, with $c_{v}$ the specific heat of the mixture at constant volume (assumed constant); the Zel'dovich number $\beta=E\left(T_{a}-T_{u}\right) / \mathcal{R} T_{a}^{2}$ or activation energy parameter; the heat release parameter $q=Q Y_{u} / c_{p} T_{u}$; the Prandtl number $\operatorname{Pr}=\nu / \mathcal{D}_{T}$, with $\nu$ the kinematic viscosity of the mixture; the Lewis number $L e=\mathcal{D}_{T} / \mathcal{D}$, with $\mathcal{D}$ the mass diffusivity of the fuel; the scaled Mach number $\mathcal{M}=\mathrm{Ma}^{2} / a^{2}$, where $\mathrm{Ma}=S_{L} / c$ is the representative Mach number corresponding to the ratio of the laminar flame speed $S_{L}$ and the speed of sound $c=\left(\gamma p_{u} / \rho_{u}\right)^{1 / 2}$ at atmospheric pressure. We note parenthetically that $\mathrm{Pr}^{-1}$ is the Reynolds number $R e$, when the latter is based on the flame thickness $\delta_{T}$ and the laminar flame speed $S_{L}$.

The analytic formula for the laminar flame speed,

$$
S_{L}^{\text {as }}=\sqrt{2 L e \beta^{-2} \mathcal{D}_{T} \mathcal{B} \rho_{u}^{n-1}}\left(T_{u} / T_{a}\right)^{n / 2} \mathrm{e}^{-E / 2 \mathcal{R} T_{a}},
$$


obtained in the limit $\beta \rightarrow \infty$, has been used when expressing the reaction rate in dimensionless form. To account for the difference between the value $S_{L}$ for a finite value of $\beta$ and its asymptotic value, and ensure that the dimensionless flame speed of a planar adiabatic (isobaric) flame for any value of $\beta$ is equal to one, the factor $s_{L}=S_{L} / S_{L}^{\text {as }}$ has been introduced in (7). This factor is the eigenvalue of the following boundary value problem

$$
\begin{aligned}
\frac{d \theta}{d \xi}=\frac{d^{2} \theta}{d \xi^{2}}+\omega, & \frac{d Y}{d \xi}=L e^{-1} \frac{d^{2} Y}{d \xi^{2}}-\omega, \\
\theta=0, \quad Y=1 & \text { as } \xi \rightarrow-\infty, \\
\theta=1, \quad Y=0 & \text { as } \xi=+\infty,
\end{aligned}
$$

with $\omega$ given by (7), and for a given finite value of $\beta$ it can be easily determined numerically [3]. Clearly, $s_{L}=1+O\left(\beta^{-1}\right)$ when $\beta \rightarrow \infty$.

The temperature inside the walls of the duct is given by $\hat{T}_{\mathrm{w}}$, while its external surface is held constant, at the same temperature $T_{u}$ as that of the given mixture. The physical properties of the solid material, i.e., the density $\rho_{\mathrm{w}}$, thermal conductivity $\lambda_{\mathrm{w}}$, and heat capacity $c_{\mathrm{w}}$, are all assumed constants, with $\mathcal{D}_{\mathrm{w}}$ the thermal diffusivity. Introducing dimensionless variables

$$
h_{\mathrm{w}}=H_{\mathrm{w}} / R, \quad \theta_{\mathrm{w}}=\left(\hat{T}_{\mathrm{w}}-T_{u}\right) /\left(T_{a}-T_{u}\right),
$$

where $H_{\mathrm{w}}$ is the wall thickness, the heat equation in the solid wall $\left(1<r<1+h_{\mathrm{w}}\right)$ takes the form

$$
\frac{\partial \theta_{\mathrm{w}}}{\partial t}=\alpha\left\{\frac{\partial^{2} \theta_{\mathrm{w}}}{\partial x^{2}}+\frac{1}{a^{2}} \frac{1}{r} \frac{\partial}{\partial r}\left(r \frac{\partial \theta_{\mathrm{w}}}{\partial r}\right)\right\}
$$

where $\alpha=\mathcal{D}_{\mathrm{w}} / \mathcal{D}_{T}$ is the solid-to-gas thermal diffusivity ratio.

Boundary conditions that reflect the symmetry of the combustion field, no slip at and no mass penetration though the solid walls, and continuity of heat fluxes at the gas-solid interface, are:

$$
\begin{array}{cc}
v=0, \quad \frac{\partial Y}{\partial r}=\frac{\partial \theta}{\partial r}=\frac{\partial u}{\partial r}=0, & \text { at } r=0, \\
u=v=0, \quad \theta=\theta_{\mathrm{w}}, \quad \frac{\partial Y}{\partial r}=0, \frac{\partial \theta}{\partial r}=a^{2} \hat{\lambda} \frac{\partial \theta_{\mathrm{w}}}{\partial r}, & \text { at } r=1, \\
\theta_{\mathrm{w}}=0, & \text { at } r=1+h_{\mathrm{w}},
\end{array}
$$

where $\hat{\lambda}=\lambda_{\mathrm{w}} / a^{2} \lambda$ is the ratio of the solid-to-gas conductivities, scaled with $a^{2}$ to express the disparity between the solid and gas properties when the parameter $a$ is small. The conditions at 
the two ends of the tube are:

$$
\begin{gathered}
u=v=0, \quad \frac{\partial p}{\partial x}=\frac{\partial \theta}{\partial x}=\frac{\partial Y}{\partial x}=0, \quad \text { at } x=0, \\
p=0, \quad \theta=0, \quad \rho=1, \quad Y=1 \quad \text { at } x=\ell,
\end{gathered}
$$

where $\ell=L / \delta_{T}$ represents the length of the duct in units of the flame thickness. In general, the problem also requires specifying initial conditions describing the ignition source but the exact nature of these conditions are unnecessary in this study, as clarified below.

\section{Narrow-gap approximation}

We consider first the limit $a^{2} \ll 1$, corresponding to a narrow duct, and expand all variables in power series of $a^{2}$, namely in the form $f=f_{0}+a^{2} f_{1}+\ldots$ for a generic function $f$. This procedure is described in detail in [2] where the adiabatic case for a rectangular channel was considered. The application to a non-adiabatic and circular duct requires only minor modifications, which are summarized below.

To leading order, Eqs. (4)-(5) in the gas phase and Eq. (8) in the solid wall, together with boundary conditions $(9)$, yield

$$
\theta_{0}=\theta_{0}(x, t), \quad Y_{0}=Y_{0}(x, t), \quad \theta_{\mathrm{w}_{0}}=\theta_{0}(x, t)\left[1-\frac{\ln r}{\ln \left(1+h_{\mathrm{w}}\right)}\right] .
$$

The momentum equations (2)-(3) simplify to

$$
\frac{\partial p_{0}}{\partial x}=\operatorname{Pr} \frac{1}{r} \frac{\partial}{\partial r}\left(r \frac{\partial u_{0}}{\partial r}\right), \quad \frac{\partial p_{o}}{\partial r}=0,
$$

and, when integrated yields $p_{0}=p_{0}(x, t)$ and $u_{0}=2 U\left(1-r^{2}\right)$, where

$$
U(x, t)=2 \int_{0}^{1} r u_{0} d r=-\frac{1}{8 \operatorname{Pr}} \frac{\partial p_{0}}{\partial x}
$$

is the mean axial velocity. The equation of state then yields $\rho_{0}=\rho_{0}(x, t)$. Integrating the continuity equation (1) with respect to $r$, provides an expression for $v_{0}(r, x, t)$ (not needed in what follows) and, when applying the boundary conditions at $r=0$ and $r=1$, results in

$$
\frac{\partial \rho_{0}}{\partial t}+\frac{\partial\left(\rho_{0} U\right)}{\partial x}=0
$$

The implication is that in a narrow tube, the mixture properties in the radial direction are nearlyuniform while the axial velocity has a parabolic profile with a mean velocity $U$ that remains to be determined. 
To the next order, Eqs. (4)-(5) simplify to

$$
\begin{aligned}
\frac{1}{r} \frac{\partial}{\partial r}\left(r \frac{\partial \theta_{1}}{\partial r}\right)= & \rho_{0}\left(\frac{\partial \theta_{0}}{\partial t}+u_{0} \frac{\partial \theta_{0}}{\partial x}\right)-\frac{\partial^{2} \theta_{0}}{\partial x^{2}}+\mathcal{M} \frac{\gamma-1}{q}\left\{\frac{\partial p_{0}}{\partial t}+u_{0} \frac{\partial p_{0}}{\partial x}+\operatorname{Pr}\left(\frac{\partial u_{0}}{\partial r}\right)^{2}\right\}+\omega_{0}, \\
& \frac{1}{L e} \frac{1}{r} \frac{\partial}{\partial r}\left(r \frac{\partial Y_{1}}{\partial r}\right)=\rho_{0}\left(\frac{\partial Y_{0}}{\partial t}+u_{0} \frac{\partial Y_{0}}{\partial x}\right)-\frac{1}{L e} \frac{\partial^{2} Y_{0}}{\partial x^{2}}-\omega_{0},
\end{aligned}
$$

where $\omega_{0}=\omega\left(\rho_{0}, \theta_{0}, Y_{0}\right)$. Using the leading order solution (11), the boundary conditions (9) simplify to

$$
\begin{gathered}
\frac{\partial \theta_{1}}{\partial r}=\frac{\partial Y_{1}}{\partial r}=0 \quad \text { at } r=0, \\
\frac{\partial \theta_{1}}{\partial r}=-\frac{b}{2} \theta_{0}, \quad \frac{\partial Y_{1}}{\partial r}=0 \quad \text { at } r=1,
\end{gathered}
$$

where $b=2 \hat{\lambda} / \ln \left(1+h_{\mathrm{w}}\right)$. Note that for thin walls $\left(H_{\mathrm{w}} \ll R\right)$, the heat loss parameter

$$
b \approx \frac{\lambda_{\mathrm{w}}}{\lambda} \frac{\delta_{T}^{2}}{H_{\mathrm{w}}} \frac{2 \pi R}{\pi R^{2}}
$$

is proportional to the ratio of the perimeter and area of the duct cross section, and inversely proportional to the wall thickness. Integrating from $r=0$ to $r=1$ and using

$$
\int_{0}^{1} r\left\{u_{0} \frac{\partial p_{0}}{\partial x}+\operatorname{Pr}\left(\frac{\partial u_{0}}{\partial r}\right)^{2}\right\} d r=0,
$$

yields equations for $\theta_{0}$ and $Y_{0}$ which, together with (12) and (13), constitute a complete system of equations for the determination of the combustion field.

Introducing $\Lambda=8 \operatorname{Pr} \mathcal{M}$ and $P=\mathcal{M} p_{0}$, and dropping the subscript 0 for simplicity of notation, the narrow-gap approximation $\left(a^{2} \ll 1\right)$ yields the following system of equations

$$
\begin{gathered}
\frac{\partial \rho}{\partial t}+\frac{\partial(\rho U)}{\partial x}=0 \\
\rho \frac{\partial \theta}{\partial t}+\rho U \frac{\partial \theta}{\partial x}=\frac{\partial^{2} \theta}{\partial x^{2}}=\frac{\gamma-1}{q} \frac{\partial P}{\partial t}+\omega-b \theta \\
\rho \frac{\partial Y}{\partial t}+\rho U \frac{\partial Y}{\partial x}-\frac{1}{L e} \frac{\partial^{2} Y}{\partial x^{2}}=-\omega \\
\frac{\partial P}{\partial x}=-\Lambda U \\
1+\gamma P=\rho(1+q \theta)
\end{gathered}
$$


and boundary conditions

$$
\begin{gathered}
U=0, \quad \partial \theta / \partial x=\partial P / \partial x=\partial Y / \partial x=0, \quad \text { at } x=0 ; \\
\theta=0, \quad \rho=1, \quad P=0, \quad Y=1 \quad \text { as } x=\ell .
\end{gathered}
$$

with $\omega=\omega(\rho, \theta, Y)$ given by (7).

Except for the heat-loss term in (15), the problem is identical to the one reported earlier for the propagation in two-dimensional narrow adiabatic channels [2]. When $\Lambda \rightarrow 0$, the deviation of the pressure from its ambient value $P=0$ and the problem reduces to the one describing the propagation of a non-adiabatic isobaric flame. The key parameter here is the reduced Mach number

$$
\Lambda=8\left(\delta_{T}^{2} / R^{2}\right) \operatorname{Pr} \mathrm{Ma}^{2}
$$

that measures the importance of compressibility effects on flame propagation. We note that $\Lambda \sim \mathrm{Ma}^{2} / R e^{2}$ is proportional to the square of the Knudsen number which, at first sight, may invalidate the continuum hypothesis when $\Lambda \sim 1$. Fortunately, most of the results presented below, including the transition from a compressibility-driven flame to an isobaric flame, correspond to small values of $\Lambda$. We have occasionally extended the computations to $\Lambda=1$ only to examine the trend exhibited by the solution when systematically increasing $\Lambda$ and better understand its mathematical structure.

\section{Traveling wave solutions}

The main objective of the present study is to examine the possible development of traveling wave solutions, namely steadily propagating flames that evolve in sufficiently long tubes $\left(L \gg \delta_{T}\right.$ ) after the initial transient due to the ignition source has faded out. Hence, the boundary conditions at the far right can be safely applied when $x \rightarrow \infty$, except when the flame is near the end of the duct. We thus seek solutions of the system (14)-(18) of the form $f\left(x-s_{c} t\right)$, corresponding to a wave traveling to the right at a constant speed $s_{c}$. Let $\xi=x-s_{c} t$, the mass conservation equation (14) reduces to

$$
\frac{d}{d \xi}\left[\rho\left(U-s_{c}\right)\right]=0
$$


and, when integrated yields $\rho\left(U-s_{c}\right)=-s_{c}$ after the conditions $U \sim 0, \rho \sim 1$ have been applied as $\xi \rightarrow \infty$. Eliminating $U$ from Eqs. (17)-(18), then yields

$$
(1+\gamma P) \frac{d P}{d \xi}=-\Lambda s_{c}(\gamma P-q \theta)
$$

with the remaining equations

$$
\begin{gathered}
-s_{c} \frac{d \theta}{d \xi}=\frac{d^{2} \theta}{d \xi^{2}}-\frac{\gamma-1}{q} s_{c} \frac{d P}{d \xi}+\omega-b \theta, \\
-s_{c} \frac{d Y}{d \xi}=\frac{1}{L e} \frac{d^{2} Y}{d \xi^{2}}-\omega, \\
1+\gamma P=\rho(1+q \theta) .
\end{gathered}
$$

The boundary conditions in the fresh mixture are

$$
P=0, \quad \theta=0, \quad \rho=1, \quad Y=1 \quad \text { as } \xi \rightarrow \infty .
$$

There is, however, a significant difference in the boundary conditions imposed behind the flame, in the burned gas, when $b=0$ or $b>0$. In both cases the fuel is completely consumed and the products eventually reach a state of rest. However, for the adiabatic case $(b=0)$ the state variables tend to constant values in the burned gas region, while for the non-adiabatic case $(b>0)$ the state of the gas must reach asymptotically the state of the fresh mixture. Hence,

$$
\left.\begin{array}{cc}
\partial Y / \partial \xi=\partial \theta / \partial \xi=\partial P / \partial \xi=0 & \text { for } \quad b=0 \\
Y=\theta=P=0 & \text { for } \quad b>0
\end{array}\right\} \quad \text { as } \xi \rightarrow-\infty .
$$

The eigenvalue problem (20)-(25) describing the propagation of compressibility-driven flames will be first addressed numerically in the next section, followed by a discussion of the asymptotic approximation for large activation energies.

We note that, for the adiabatic case, the combination of Eqs. (21) and (22) leads to an equation that can be integrated once to give

$$
-s_{c}(\theta+Y)=\frac{d \theta}{d \xi}+\frac{1}{L e} \frac{d Y}{d \xi}-\frac{\gamma-1}{q} s_{c} P-s_{c},
$$

where the conditions in the fresh mixture, i.e., as $\xi \rightarrow \infty$, have been used. Together with (20) and (18) it determines the state of the burned gas, where $Y=0$, as

$$
P \sim q, \quad \theta \sim \gamma, \quad \rho \sim 1 \quad \text { as } \xi \rightarrow-\infty
$$


The next terms in the asymptotic behavior for large negative $\xi$ can be obtained by linearizing equations (20) and (26). One finds

$$
\begin{aligned}
P & \sim q+C_{1} e^{\lambda_{1} s_{c} \xi}+C_{2} e^{\lambda_{2} s_{c} \xi} \\
\theta & \sim \gamma+C_{3} e^{\lambda_{1} s_{c} \xi}+C_{4} e^{\lambda_{2} s_{c} \xi}
\end{aligned}
$$

where the $C_{i}$ 's are constants of integration and

$$
\lambda_{1,2}=\frac{-(1+\Lambda \gamma+q \gamma) \pm \sqrt{(1+\Lambda \gamma+q \gamma)^{2}-4 \Lambda(1+q \gamma)}}{2(1+q \gamma)} .
$$

Since $\lambda_{1,2}<0$, the solution behind the flame for the adiabatic case decays exponentially towards the equilibrium values (27). These values, reached when the reaction rate becomes negligibly small, determine the flame pressure, temperature and density (denoted by the subscript $f$ ), namely $P_{f}=q, \theta_{f}=\gamma$ and $\rho_{f}=1$.

The state of the burned gas and the values of $P_{f}, \theta_{f}, \rho_{f}$ for the non-adiabatic case $(b \neq 0)$, remain to be determined.

\section{Numerical results}

The calculations reported below were carried out in a finite domain, $\xi_{\min }<\xi<\xi_{\max }$, which was increased systematically in order to determine the independence of the results to the computational domain. The spatial derivatives were discretized on a uniform grid using a second order central difference scheme and the equations were solved using a Gauss-Seidel method with over-relaxation. The number of grid points was doubled in some cases without notable difference in the results. The solutions were obtained using two types of iterations. When a single-valued response was expected, e.g., when $b=0$, the value of the flame velocity $s_{c}$ was calculated directly as a function of parameters. In the presence of heat loss a multivalued response was anticipated, and the value of $b$ was calculated for given $s_{c}$. In either case, a Gauss-Seidel procedure was used to compute $s_{c}$ or $b$.

In order to eliminate the freedom in shifting the coordinate $\xi$ by a constant, we have fixed the temperature at an internal point within the domain; say $\theta=\theta_{*}$ at $\xi=\xi_{*}$, with $\theta_{*}$ chosen judiciously. The choice $\theta_{*}=0.8 \div 1.1$ was found appropriate in all the reported calculations. The value $\theta_{*}$ is shown on the temperature profiles of Fig. 2 reported below, with an open circle. 
To render the extensive computations manageable, the boundary conditions (25) were conveniently replaced with the "mild" conditions

$$
\frac{\partial^{2} P}{\partial \xi^{2}}=\frac{\partial^{2} \theta}{\partial \xi^{2}}=\frac{\partial^{2} Y}{\partial \xi^{2}}=0 \quad \text { as } \xi \rightarrow-\infty .
$$

which permits reducing the size of the integration domain. For the adiabatic case, these conditions are automatically satisfied. For the non-adiabatic case, we have verified that using (5) instead of (25) has a negligible influence on the solution when the size of the computational domain is sufficiently large.

In most of the calculations reported below we have chosen $q=5, \gamma=1.4$ and $\beta=10$, focusing on variations in the heat loss parameter $b$, the Lewis number $L e$, and the compressibility parameter $\Lambda$. In order to examine the importance of the exponent $n$ on flame propagation, the two values $n=1$ and $n=2$ have been examined.

\subsection{Adiabatic flames}

We consider first the adiabatic duct, $b=0$, which was previously examined in [2] for the case $n=2$. Profiles of the state variables across the flame are shown in Fig. 1 for the two exponents $n=1$ and $n=2$ and for $\Lambda=1$ and $\Lambda=0.1$, with a magnification of the region near the reaction zone presented in Fig. 2 for $\Lambda=0.1$. In the latter the reference temperature $\theta_{*}$ used in the computations is marked by the symbol $\circ$. We observe that the flame structure is remarkably different than the structure of ordinary isobaric flames. The preheat zone is preceded by a region where the gas is heated by adiabatic compression, with the pressure and density increasing continuously. This region, corresponding to $Y \approx 1$, is followed by a relatively thin convective-diffusive zone, where the temperature increases abruptly by the heat conducted from the reaction zone with the density dropping sharply as a result of gas expansion, and a much thinner diffusive-reactive layer where combustion occurs with the fuel being completely depleted $(Y \rightarrow 0)$. The thickness of the reaction zone is shown in Fig. 2 using the spatial distribution of the reaction rate $\omega$. As a result of the heat released by the chemical reactions and the continuous increase in pressure, the temperature rises above the isobaric adiabatic flame temperature to the value $\theta_{f}=\gamma$, corresponding to the isochoric adiabatic flame temperature, with the density dropping to $\rho_{f}=1$. The pressure attains its maximum value $P_{f}=q$ in the reaction zone. The state of the gas behind the flame remains constant, with all variables retaining their values reached in the reaction zone. 


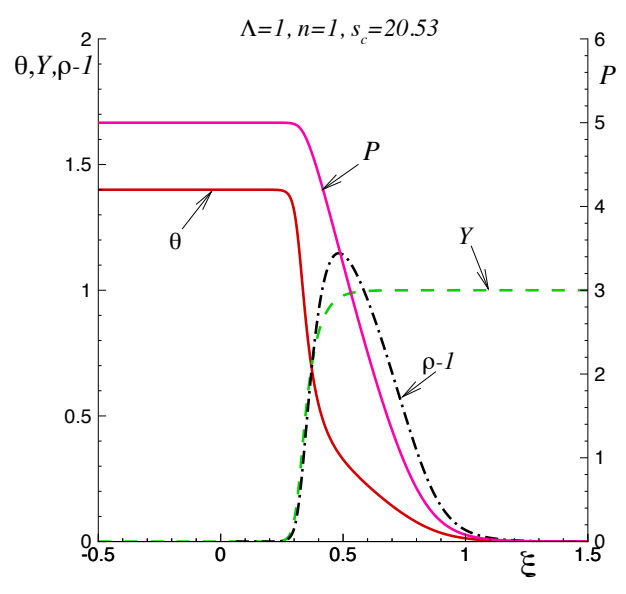

(a) $\Lambda=1, n=1, s_{c}=20.53$

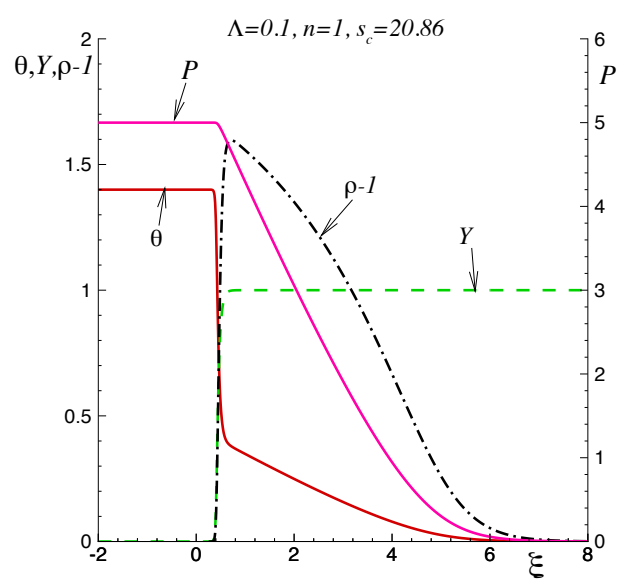

(c) $\Lambda=0.1, n=1, s_{c}=20.86$

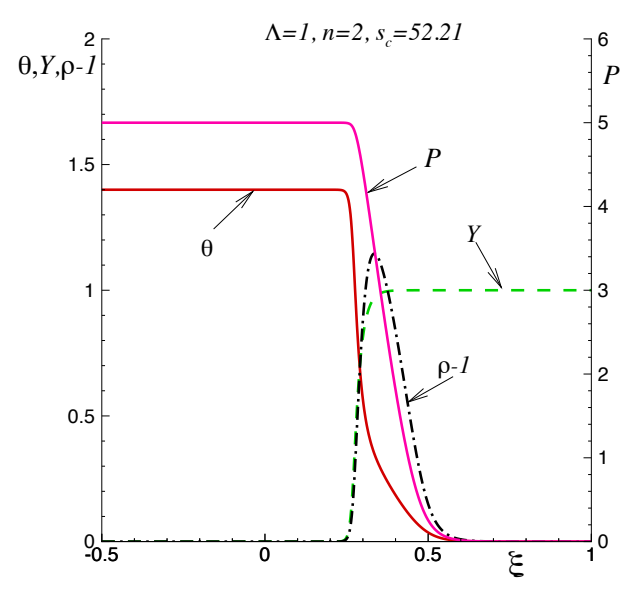

(b) $\Lambda=1, n=2, s_{c}=52.21$

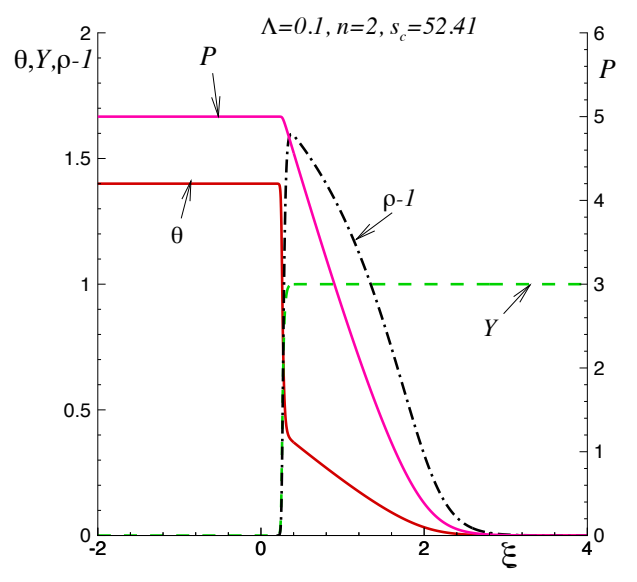

(d) $\Lambda=0.1, n=2, s_{c}=52.41$

Figure 1: Pressure, temperature, density, and mass fraction $Y$ profiles of adiabatic $(b=0)$ compressibility-driven flames with $L e=1$, for two values of the compressibility parameter $\Lambda$ and the two exponents $n=1$ and $n=2$; the unburned gas on the right, and the burned gas on the left sides. In each case the propagation speed $s_{c}$ is indicated. 


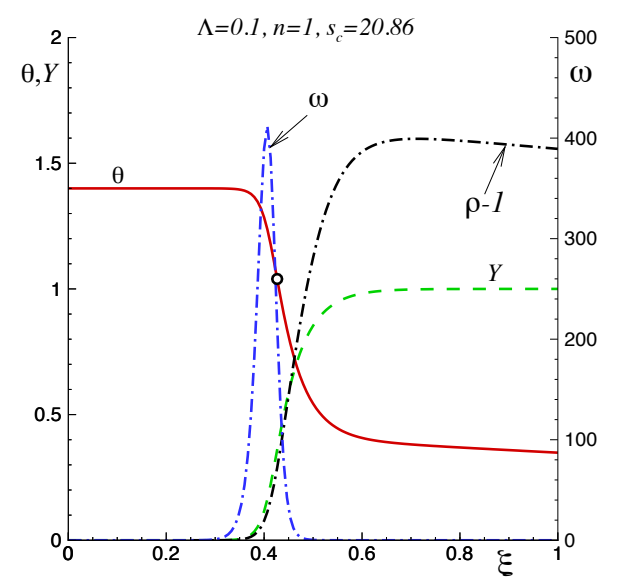

(a) $n=1$

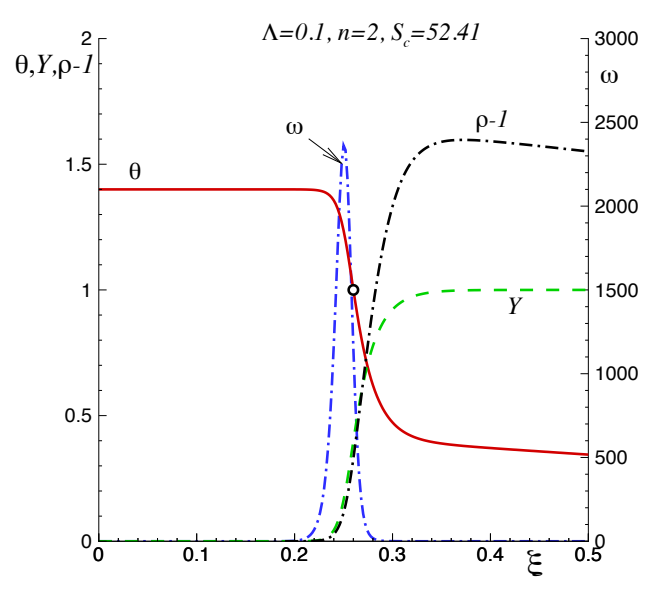

(b) $n=2$

Figure 2: Magnification of the temperature, density and mass fraction profiles of the adiabatic $(b=0)$ compressibilitydriven flame with $\Lambda=0.1$ shown in Fig. 1 in the vicinity of the reaction zone; the reference point marked with a symbol o correspond to $\theta_{*}$.

Due to the relatively slow preheating caused by adiabatic compression when $\Lambda$ is small, the overall flame zone is much thicker. The increase in temperature and corresponding drop in density across the reaction zone appear much sharper and occur in a relatively thinner region. This "jump" in density and temperature across the reaction zone is in sharp contrast to the continuous change observed in isobaric flames. It would have been instructive, therefore, to examine the flame structure by further reducing the value of $\Lambda$. Unfortunately, due to the steepening of the temperature and density profiles and the widening of the preheat zone, serious numerical difficulties are encountered when attempting to find solutions with a distributed reaction rate for smaller values of $\Lambda=0.1$.

The propagation speed as a function of $\Lambda$, for unity, sub- and super- unity Lewis number flames, is plotted in Fig. 3 for the two values $n=1$ and $n=2$. As discussed in [2], the propagation speed of compressibility-driven flames is much larger than the speed of isobaric flames; the influence of compressibility manifests itself though the increase in flame temperature from $\theta_{f}=1$ to $\theta_{f}=\gamma$, amplified by the large activation energy of typical combustion reactions. The relatively weak dependence of $s_{c}$ on the Lewis number is expected, because of the secondary 


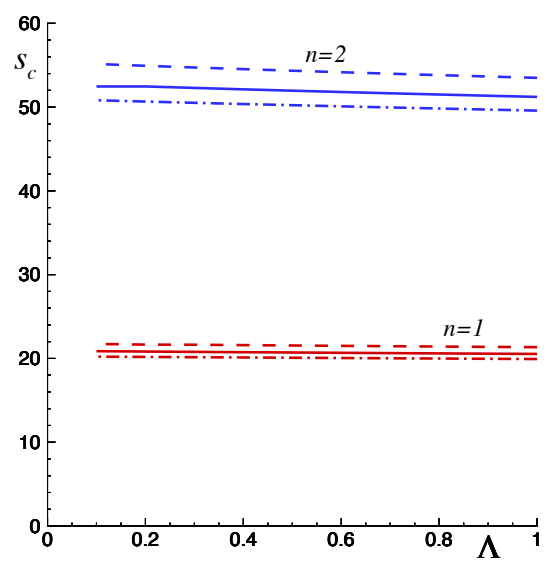

Figure 3: The propagation speed of an adiabatic compressibility-driven flame as a function of the compressibility parameter $\Lambda$, for $n=1$ and 2 and three values of the Lewis numbers: $L e=1.5$ - dash-dotted lines, $L e=1$ - solid lines $L e=0.5$ - dashed lines.

role that thermo-diffusive effects have on the propagation of planar flames. The notable increase in $s_{c}$ when increasing the exponent $n$ is due to the large variations in density, particularly near the reaction zone, which has a direct effect on the reaction rate. The most surprising result is the lack of dependence of the propagation speed on the compressibility parameter, which persists even when decreasing $\Lambda$ to small values, and does not approach the expected value $s_{c}=1$ when $\Lambda=0$. Evidently, the limit $\Lambda \rightarrow 0$ is singular, as is also observed from the structure of the solution shown in Fig. 1 when reducing $\Lambda$. Since the classical isobaric formulation is recovered when formally imposing $\Lambda=0$ in the governing equations (20)-(24), treating the case $\Lambda \ll 1$ requires taking the analysis to higher orders, which is beyond the scope of the present article. The limit $\Lambda \rightarrow 0$ will be clarified in the following by numerically investigating the propagation of non-adiabatic flames and systematically reducing the values of $\Lambda$ and the heat loss parameter $b$.

\subsection{Non-adiabatic flames}

It is well known, for isobaric flames, that the dependence of the propagation speed on the heat-loss parameter is multi-valued for $0 \leq b<b_{c}$, and no (steady) solution exist for $b>b_{c}$. The response curve of $s_{c}$ vs $b$ is therefore C-shaped, with a turning point at $b=b_{c}$ corresponding to flame extinction. The upper branch, along which the propagation speed decreases from 
$s_{c}=1$ when increasing the heat loss parameter $b$, corresponds to stable states; the lower branch along which $s_{c}$ increases with increasing $b$ corresponds to unstable states that cannot be realized physically. The critical parameter $b_{c}$ is given in the asymptotic limit $\beta \rightarrow \infty$ by $b_{c}=(\beta \mathrm{e})^{-1}$ $[4,5]$, a result that has been recently extended in [6] to large but finite values of $\beta$.

The response curve tracing the propagation speed as a function of the heat loss parameter is also C-shaped, as shown in Fig. 4 for several values of $\Lambda$ and the two exponents $n=1$ and $n=2$. The focus presently should be on the solid curves, which were obtained numerically; the dashed curves representing the asymptotic approximation for large $\beta$ will be discussed in the next section. For $0 \leq b<b_{c}$ the solution is multi-valued. The two solutions for a given $b<b_{c}$ are quite distinct as illustrated in Fig. 5, where temperature, pressure and fuel mass fraction profiles are plotted for each of the two solutions corresponding to $b=1.31$ (the two steady states are marked in Fig. 4(a) by the symbol $\circ$ ). Due to heat loss, the flame temperature $\theta_{f}<\gamma$ and the pressure $P_{f}<q$ in both cases, but the flame associated with a higher temperature and pressure propagates nearly three times faster than the the flame with the lower temperature and pressure. Steady states along the upper branch of the response curve, corresponding to solutions with $s_{c}$

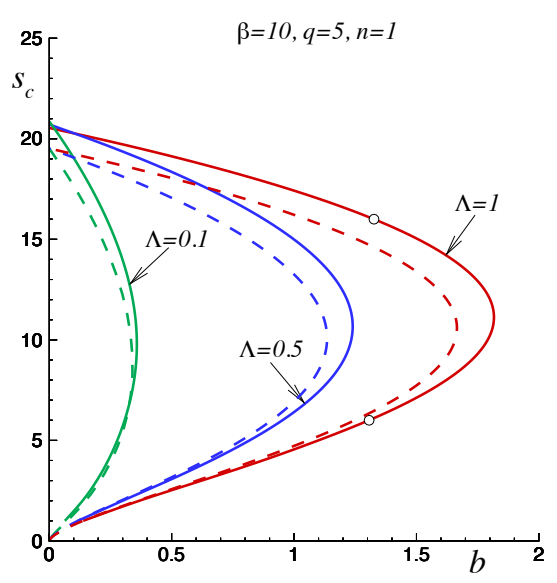

(a) $n=1$

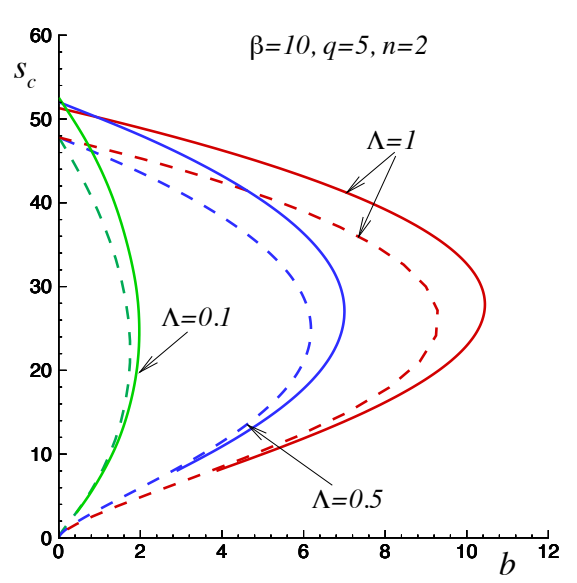

(b) $n=2$

Figure 4: Propagation speed of compressibility-driven flames as a function of the heat loss parameter $b$ for $O(1)$ values of the compressibility parameter $\Lambda$ and $L e=1$; the solid lines correspond to the numerical results with $\beta=10$, the dashed lines are based on the asymptotic approximation derived in Sec. 6. 


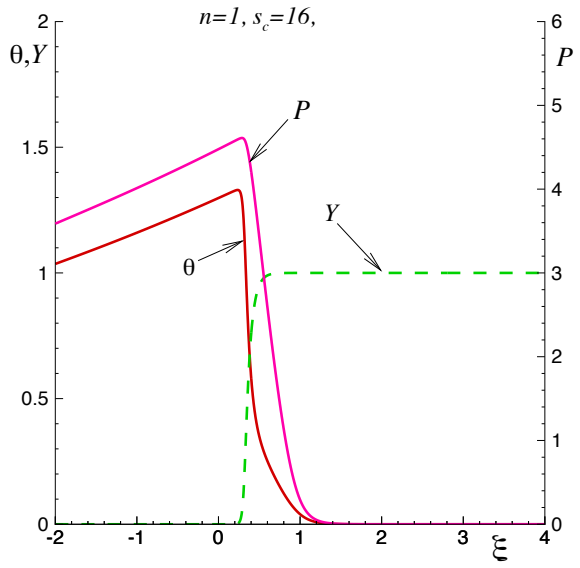

(a) Solution corresponding to $s_{c}=16$

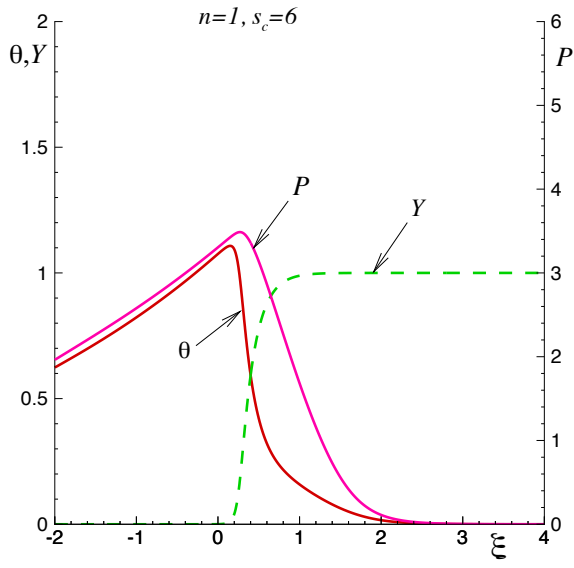

(b) Solution corresponding to $s_{c}=6$

Figure 5: Profiles of temperature, pressure and mass fraction of compressibility-driven flames corresponding to the two distinct solutions for $b \approx 1.31$, marked in Fig. 4(a) by the symbol ॰; computed for $\Lambda=1$ and $n=1$.

that decrease from the adiabatic value when increasing $b$, are presumably stable; those along the lower branch of the response curve with $s_{c}$ increasing when increasing $b$ are unstable. One notes that the critical heat-loss parameter $b_{c}$ for compressibility-driven flames is much larger than the corresponding value for isobaric flames, implying that these fast flames can resist heat-loss to a greater extent without being extinguished. Evidently, $b_{c}=O(1)$ even for the relatively large value of $\beta=10$ considered here, and depending on $\Lambda$ and $n$ can be as large as $b=10$.

\section{Asymptotic analysis}

In this section, we derive an asymptotic solution of the eigenvalue problem (20)-(24) for large activation energy $\beta$, that more clearly elucidates the structure of compressibility-driven flames and the dependence of their propagation speed on the relevant parameters. In particular, we are interested in the dependence of $s_{c}$ ranging from $\mathrm{O}(1)$ to small values of $\Lambda$, to clarify the limiting behavior $\Lambda \rightarrow 0$. It is convenient to recast the exponential term in the reaction-rate (7) using

$$
\exp \left\{\frac{\beta(\theta-1)}{1+q(\theta-1) /(1+q)}\right\}=\mathrm{e}^{A} \exp \left\{\frac{\tilde{\beta}\left(\theta-\theta_{f}\right)}{1+\tilde{q}\left(\theta-\theta_{f}\right) /(1+\tilde{q})}\right\},
$$


where $\theta_{f}$ remains to be specified,

$$
A=\frac{\beta\left(\theta_{f}-1\right)}{1+q\left(\theta_{f}-1\right) /(1+q)}, \quad \tilde{q}=\frac{q}{1+q\left(\theta_{f}-1\right) /(1+q)},
$$

and the activation energy parameter is expressed in the form

$$
\beta=\left[1+q\left(\theta_{f}-1\right) /(1+q)\right]^{2} \tilde{\beta} .
$$

Evidently, $\tilde{\beta}$ and $\beta$ have the same order of magnitude, and $\tilde{\beta} \rightarrow \infty$ when $\beta \rightarrow \infty$. The reaction rate becomes

$$
\omega=\underbrace{\frac{(1+q)^{n} \rho_{f}^{n}}{s_{c}^{2}}\left(\frac{\beta}{\tilde{\beta}}\right)^{2} \mathrm{e}^{A}}_{\mu} \frac{\tilde{\beta}^{2} s_{c}^{2}}{2 L e s_{L}^{2}}\left(\frac{\rho}{\rho_{f}}\right)^{n} Y \exp \left\{\frac{\tilde{\beta}\left(\theta-\theta_{f}\right)}{1+\tilde{q}\left(\theta-\theta_{f}\right) /(1+\tilde{q})}\right\} .
$$

A similar transformation was used in [2] to study the structure of adiabatic compressibility-driven flames and in [6] to generalize the asymptotic results for non-adiabatic isobaric flames to large but finite values of $\beta$.

For large $\tilde{\beta}$ the chemical reaction is confined to a sheet along which $\theta=\theta_{f}$, such that $\theta_{f}$ stands for the flame temperature. If the pressure and density along the sheet are denoted by $P_{f}$ and $\rho_{f}$, then $\rho_{f}=\left(1+\gamma P_{f}\right) /\left(1+q \theta_{f}\right)$. Under adiabatic conditions, $\theta_{f}=\gamma, P_{f}=q$ and $\rho_{f}=1$, but for non-adiabatic flames these values remain to be determined. Finally, we note that in numerical calculations with a distributed reaction, i.e., with finite $\tilde{\beta}$, an appropriate choice for the determination of the flame temperature, density and pressure is the location where the reaction rate takes its maximum value.

Rescaling length using $\zeta=s_{c} \xi$, the governing equations (20)-(22) become

$$
\begin{gathered}
(1+\gamma P) \frac{d P}{d \zeta}=-\Lambda(\gamma P-q \theta) \\
-\frac{d \theta}{d \zeta}=\frac{d^{2} \theta}{d \zeta^{2}}-\frac{\gamma-1}{q} \frac{d P}{d \zeta}+\tilde{\omega}-\tilde{b} \theta \\
-\frac{d Y}{d \zeta}=\frac{1}{L e} \frac{d^{2} Y}{d \zeta^{2}}-\tilde{\omega},
\end{gathered}
$$

where

$$
\tilde{\omega}=\mu \frac{\tilde{\beta}^{2}}{2 \operatorname{Les}_{L}^{2}}\left(\frac{\rho}{\rho_{f}}\right)^{n} Y \exp \left\{\frac{\tilde{\beta}\left(\theta-\theta_{f}\right)}{1+\tilde{q}\left(\theta-\theta_{f}\right) /(1+\tilde{q})}\right\} .
$$

Here $\mu$ is the effective Damköhler number given by

$$
\mu=\frac{1}{s_{c}^{2}}\left(1+\gamma P_{f}\right)^{n}\left(\frac{1+q}{1+q \theta_{f}}\right)^{n-4} \mathrm{e}^{A},
$$


and $\tilde{b}=b s_{c}^{-2}$ plays the role of a parameter. For a specified $\tilde{b}$, the solution of Eqs. (31)-(33) together with the boundary conditions (24)-(25) determines the values of $\theta_{f}, P_{f}, \rho_{f}$ as well as $\mu$. The latter then provides the following expression for the propagation speed,

$$
s_{c}=\mu^{-1 / 2}\left(1+\gamma P_{f}\right)^{n / 2}\left(\frac{1+q \theta_{f}}{1+q}\right)^{2-n / 2} \exp \left\{\frac{\beta}{2} \frac{\theta_{f}-1}{\left(1+q \theta_{f}\right) /(1+q)}\right\},
$$

with the corresponding heat loss parameter obtained a-posteriori from

$$
b=\tilde{b} s_{c}^{2} .
$$

The formulation, so far, is valid for any value of $\tilde{\beta}$, but will be used below to determine an asymptotic approximation to the propagation speed, denoted as $s_{c}^{\text {as }}$, valid for $\tilde{\beta} \gg 1$.

\subsection{Outer zones}

We proceed with the asymptotic solution for $\tilde{\beta} \gg 1$. Chemical reaction in this limit is confined to a sheet which, without loss of generality, may be located at $\zeta=0$. Elsewhere, in the pre- and post-reaction regions, or the outer regions where $\zeta>0$ and $\zeta<0$ respectively, the reaction rate is exponentially small and the problem reduces to solving (31)-(33) with $\tilde{\omega}=0$. Across the reaction front all variables remain continuous, namely $\llbracket P \rrbracket=\llbracket \theta \rrbracket=\llbracket Y \rrbracket=0$, where the operator $\llbracket f \rrbracket=\left.f\right|_{\zeta=0^{+}}-\left.f\right|_{\zeta=0^{-}}$denotes the jump in the quantity $f$ across $\zeta=0$. Since the reaction rate can be eliminated when adding Eqs. (32) and (33), the additional jump condition

$$
\llbracket \frac{d \theta}{d \zeta} \rrbracket+\frac{1}{L e} \llbracket \frac{d Y}{d \zeta} \rrbracket=0,
$$

is obtained when integrating the resulting equation across $\zeta=0$. The solution for the fuel mass fraction in the outer regions is given by

$$
Y= \begin{cases}0, & \zeta<0, \\ 1-\exp (-L e \zeta), & \zeta>0 .\end{cases}
$$

The solution for the pressure and temperature requires solving

$$
\begin{aligned}
& (1+\gamma P) \frac{d P}{d \zeta}=-\Lambda(\gamma P-q \theta), \\
& -\frac{d \theta}{d \zeta}=\frac{d^{2} \theta}{d \zeta^{2}}-\frac{\gamma-1}{q} \frac{d P}{d \zeta}-\tilde{b} \theta,
\end{aligned}
$$

subject to the jump conditions

$$
\llbracket P \rrbracket=\llbracket \theta \rrbracket=0, \quad \underset{18}{\llbracket d \theta / d \zeta \rrbracket=-1}
$$


across the reaction sheet, and the boundary conditions $P \sim 0$ and $\theta \sim 0$ as $\zeta \rightarrow \pm \infty$. The jump and boundary conditions are sufficient for determining the solution ahead and behind the reaction front. However, it is a nonlinear problem that does not possess an exact analytical solution, and has been solved numerically using a shooting method, as described in Appendix A.

Figure 6 shows typical profiles of the temperature (red/solid lines) and pressure (blue/dashed lines) in the outer regions, calculated for $q=5$. The sharp increase in temperature ahead of the flame compared to slow decrease behind the flame is in agreement with the numerical results
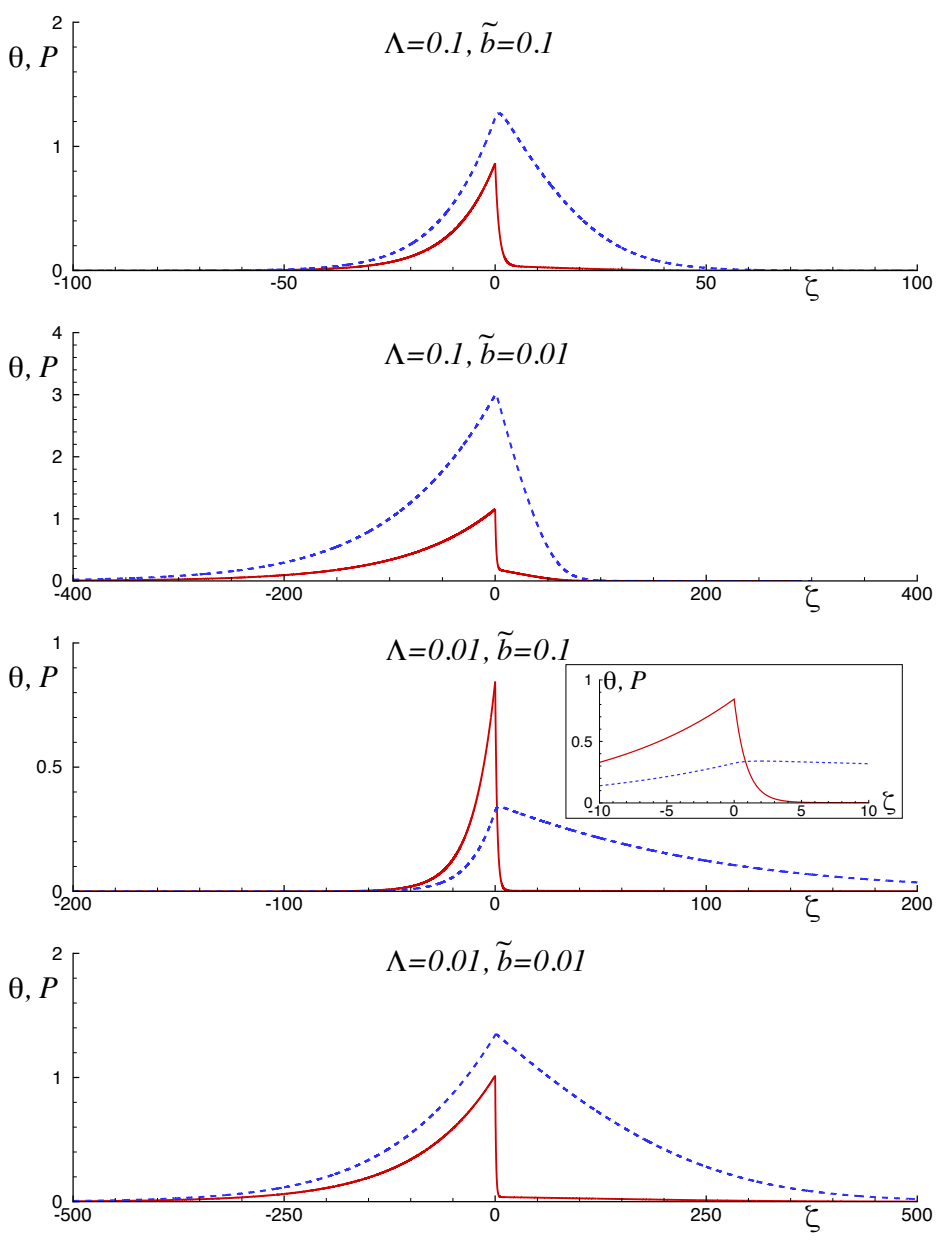

Figure 6: Profiles of temperature (red/solid lines) and pressure (blue/dashed lines) in the pre- and post-reaction front (outer solutions) of compressibility-driven flames, determined from the asymptotic solution for large $\tilde{\beta}$ with $q=5$. 
reported in Fig. 5. The jump in the temperature derivative across the reaction zone $(\zeta=0)$ is more clearly seen in the rescaled region shown in the figure inset.

Figure 7 shows the calculated values of the flame temperature $\theta_{f}$ and pressure $P_{f}$ as a function of the (scaled) heat loss parameter $\tilde{b}$, for a wide range of $\Lambda$. Both, $\theta_{f}$ and $P_{f}$, tend to their adiabatic values, $\theta_{f}=\gamma$ and $P_{f}=q$, when $b \rightarrow 0$, and decrease substantially in the presence of heat loss. For a given value of $\tilde{b}$, the flame temperature and pressure decrease when decreasing $\Lambda$, but not much when when $\tilde{b}$ exceeds a certain threshold. Finally, we note that we have deliberately selected in this figure values of $\Lambda<0.1$, to demonstrate that the asymptotic solution captures a range of parameters where the direct numerical computations faced some difficulties.

In Fig. 8 we show the temperature gradient $\Phi \equiv d \theta /\left.d \zeta\right|_{\zeta=0^{-}}$, which corresponds to the amount of heat conducted to the burned gas region from the reaction zone, as a function of $\tilde{b}$. In the adiabatic case, all the heat generated in the reaction zone is conducted to the fresh mixture; the temperature of the burned gas remains constant, i.e., $\theta=\theta_{f}=\gamma$, and $\Phi=0$. In the presence of heat loss, the temperature of the burned gas drops below $\theta_{f}$, with $\Phi$ increasing when increasing $\tilde{b}$. The graph also shows that the heat conducted to the burned gas is primarily a result of heat

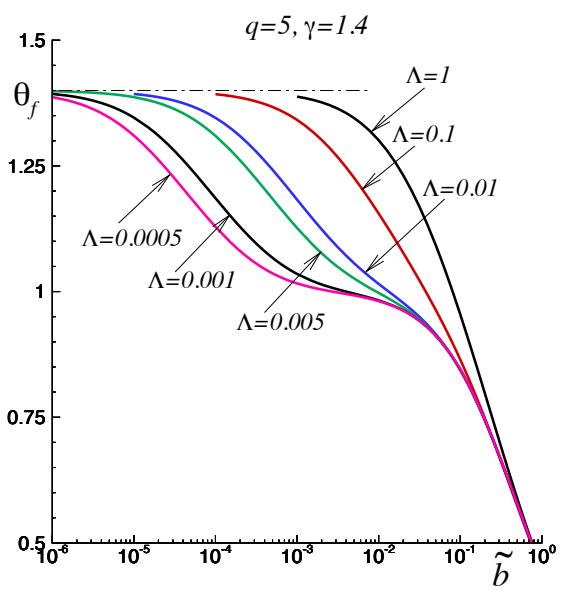

(a) Flame temperature $\theta_{f}$

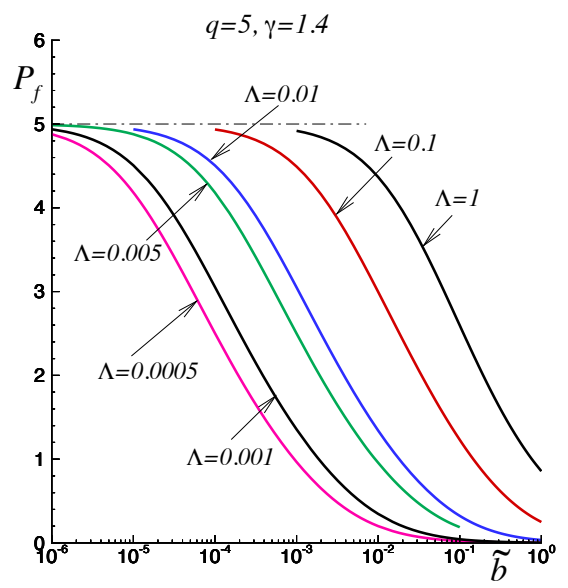

(b) Flame pressure $P_{f}$

Figure 7: Flame temperature and pressure of compressibility-driven flames, plotted as a function of $\tilde{b}$ for various values of $\Lambda$; determined from the asymptotic solution for large $\tilde{\beta}$ with $q=5$ and $\gamma=1.4$. 


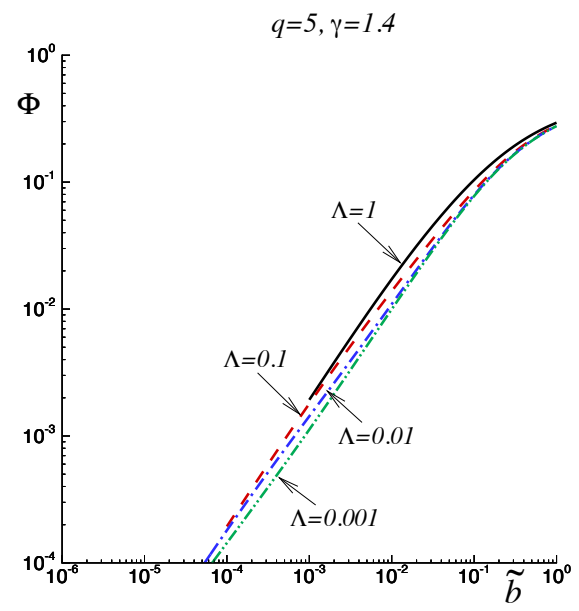

Figure 8: The temperature gradient $\Phi=d \theta /\left.d \zeta\right|_{\zeta=0^{-}}$, corresponding to the heat conducted to the burned gas region; calculated for various $\Lambda$ with $q=5$ and $\gamma=1.4$.

loss and depends only weakly on the compressibility parameter $\Lambda$. In the range $0<\Lambda<1$, it may be treated as practically independent of $\Lambda$. Finally, we note that the value of $\Phi$ is needed in the analysis of the reaction zone discussed next.

\subsection{Reaction zone}

Although the outer solutions provided the pressure, temperature and fuel mass fraction distributions across the flame, the details of the reaction zone are needed for the determination of the propagation speed. To this end, we introduce the stretching transformation, $\zeta=\tilde{\beta}^{-1} \eta$, and expand all variables about their values at the reaction front, namely

$$
P=P_{f}-\tilde{\beta}^{-1} \chi(\eta)+\ldots, \quad \theta=\theta_{f}-\tilde{\beta}^{-1} \phi(\eta)+\ldots, \quad Y=\tilde{\beta}^{-1} \psi(\eta)+\ldots
$$

Substituting into the governing equations (31)-(33) yields

$$
\frac{d \chi}{d \eta}=0, \quad \frac{d^{2} \phi}{d \eta^{2}}=\frac{1}{2 L e} \mu \psi \mathrm{e}^{-\phi}, \quad \frac{1}{L e} \frac{d^{2} \psi}{d \eta^{2}}=\frac{1}{2 L e} \mu \psi \mathrm{e}^{-\phi},
$$

where the leading terms $s_{L} \sim 1$ and $\rho \sim \rho_{f}$ have been used in evaluating the reaction rate $\tilde{\omega}$. The first equation indicates that $\chi \equiv 0$ and verifies that the pressure, to leading order, remains continuous across the reaction zone. Matching the inner and outer expansions requires

$$
\psi=\frac{d \psi}{d \eta} \sim 0, \quad \frac{d \phi}{d \eta} \underset{21}{\sim-\Phi,} \quad \text { as } \eta \rightarrow-\infty
$$




$$
\frac{1}{L e} \frac{d \psi}{d \eta} \sim 1 \quad \frac{d \phi}{d \eta} \sim-\Phi+1, \quad \text { as } \eta \rightarrow+\infty
$$

which are used as boundary conditions for the determination of $\phi(\eta)$ and $\psi(\eta)$.

The first integral of Eqs. (41) yields

$$
\frac{d \phi}{d \eta}-\frac{1}{L e} \frac{d \psi}{d \eta}=-\alpha
$$

and, when using the matching condition as $\eta \rightarrow+\infty$, one finds that $\alpha=\Phi$, a constant that has been already determined by the outer solution, as shown in Fig. 8. A second integration yields

$$
\phi-\frac{1}{L e} \psi=-\alpha \eta
$$

where, without loss of generality, the constant of integration has been set to zero by appropriately choosing the origin of the stretched coordinate $\eta$. Let $f=L e^{-1} \psi$ the inner problem reduces to solving

$$
\begin{gathered}
\frac{d^{2} f}{d \eta^{2}}=\frac{1}{2} \mu f \mathrm{e}^{-f+\alpha \eta} \\
f=\frac{d f}{d \eta} \sim 0 \quad \text { as } \eta \rightarrow-\infty, \quad f \sim \eta \quad \text { as } \eta \rightarrow+\infty
\end{gathered}
$$

Equation (42) with the three conditions (43) can be solved numerically, for given $\alpha$, to determine $f(\eta)$ and the constant $\mu$. This boundary value problem was previously encountered when examining the structure of diffusion flames in the "premixed flame regime" [7], and was recently reexamined in [6]. Rather than fitting the numerical data to determine the dependence of $\mu$ on $\alpha$, we use below an approximation valid for $\alpha \ll 1$. When $\alpha=0$, corresponding to the adiabatic case, eq. (42) can be integrated once and, when applying the boundary conditions (43) yields that $\mu=1$. A standard regular perturbation method yields the next two terms, such that

$$
\mu=1-\mu_{1} \alpha-\mu_{2} \alpha^{2}+\ldots,
$$

with $\mu_{1} \approx 1.3440$ and $\mu_{2} \approx-0.9032$.

In summary, the outer solution determines the profiles of the temperature, pressure and fuel mass fraction, as well as the values $\theta_{f}, P_{f}$ and $\Phi$ as function of $\tilde{b}$, and the inner solution provides, for a given $\Phi$, the value of $\mu$. The asymptotic approximation of the flame speed, $s_{c}^{\text {as }}$, is then obtained from Eq. (34) with the corresponding heat loss parameter obtained from (35). Finally, we note that for the adiabatic case $(\mu=1)$ the asymptotic approximation $s_{c}^{\text {as }}$ reduces to the expression derived in [2] for $n=2$. 


\subsection{Comparison to the numerical results}

Next, we compare the asymptotic approximation to the propagation speed, $s_{c}^{\text {as }}$, to the numerical solution $s_{c}$ obtained directly from (20)-(24). Considering first the adiabatic case, we show in Fig. 9 the numerically-computed $s_{c}$, plotted for $\Lambda=1$ (solid lines) and $\Lambda=0.2$ (marked with a $\triangle$ symbol), as a function of the Zel'dovich number $\beta$, for the two exponents $n=1$ and $n=2$. Also shown in the figure is $s_{c}^{\text {as }}$ (dashed lines) which, as noted earlier, is practically independent of $\Lambda$. Evidently, the asymptotic result approximates well the "exact" propagation speed computed numerically for a wide range of $\beta$. The difference between the two, however, does not seem to reduce appreciably when increasing $\beta$, as may have been expected. The reason is due to the exponentially growing factor in the asymptotic expression for $s_{c}$, as discussed in Appendix B.

In Fig. 4 we have added the dependence of $s_{c}^{\text {as }}$ on $b$, for the corresponding values of $\Lambda$ and $n$, as dashed curves. The comparison shows that the asymptotic expression approximates the "exact" numerical results extremely well. The minor discrepancies result from adopting

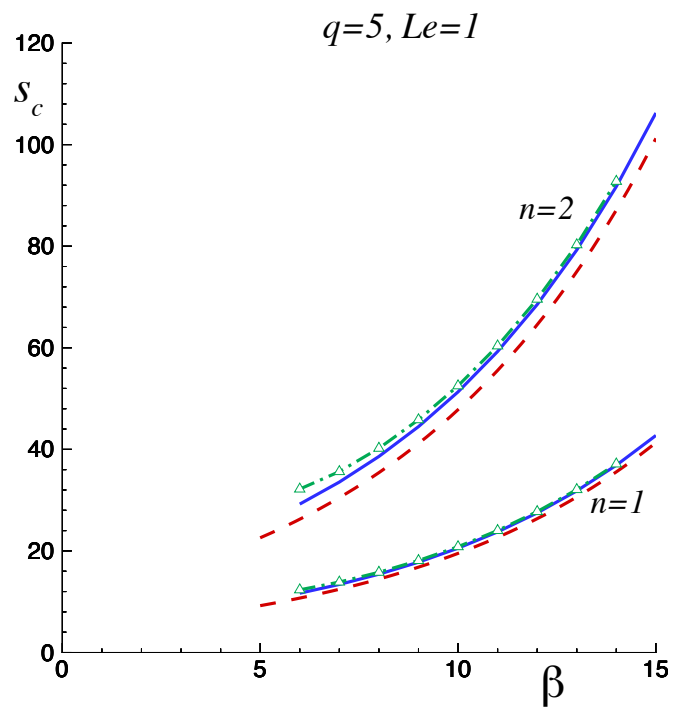

Figure 9: Comparison of the propagation speed of adiabatic, compressibility-driven flames $s_{c}$ determined numerically (solid lines for $\Lambda=1$ and triangle symbols for $\Lambda=0.2$ ) to the asymptotic approximation $s_{c}^{\text {as }}$ (dashed lines) for the two exponents $n=1$ and $n=2$, with $q=5$ and $L e=1$. 
the approximate expression (44) for $\mu$ and retaining only the leading term in the inner/outer expansions.

\section{The transition from fast to slow flames}

Using the asymptotic approximation derived in the preceding section, we now examine the propagation speed of compressibility-driven flames for small values of $\Lambda$, which was found prohibitively difficult to evaluate numerically. In Fig. 10 we present response curves of $s_{c}^{\text {as }}$ versus $b$, for values of $\Lambda$ that systematically approach zero. The dashed curve corresponds to $\Lambda=0$, or isobaric flames. The upper branch of all curves with $\Lambda>0$ terminates at the same point when $b=0$, which corresponds to the propagation speed of adiabatic flames (as discussed earlier, this value is practically independent of $\Lambda$ ) and is marked in the figure with the symbol $\bullet$; the upper branch of the dashed curve terminates at $s_{c}^{\text {as }}=1$. The graphs in this figure demonstrate the transition that occurs in the $\mathrm{C}$-shaped response curve when reducing the compressibility parameter. For sufficiently small values of $\Lambda$ the response curve for a given value of $b$ consists of four distinct solutions, instead of two, as clearly illustrated in the figure insets. Assuming that

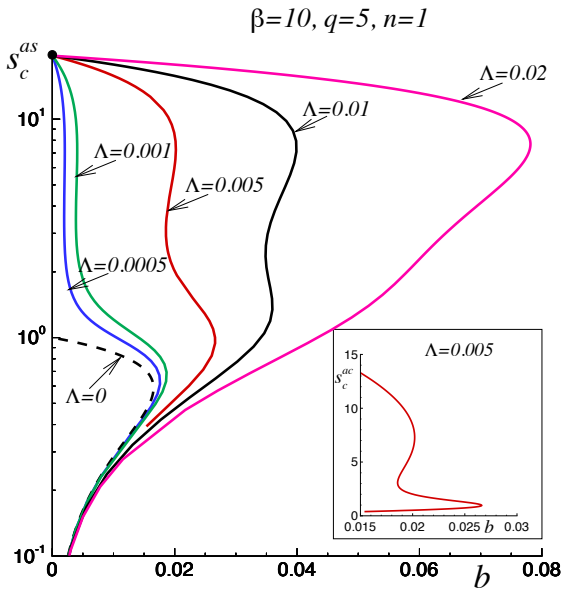

(a) $n=1$

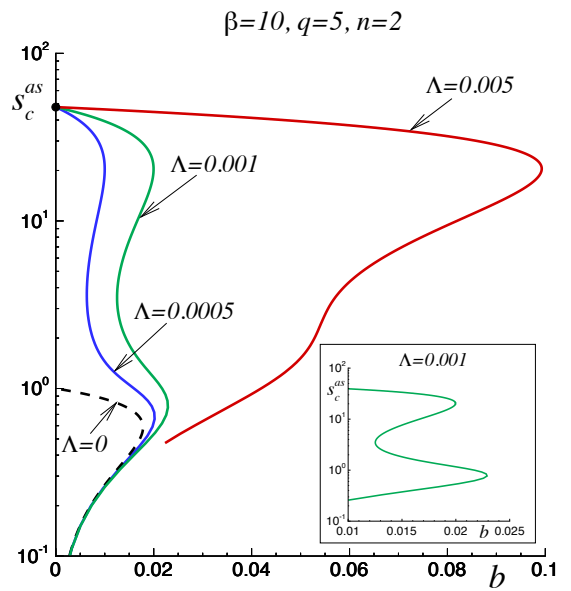

(b) $n=2$

Figure 10: Propagation speed (based on the asymptotic approximation with $\beta=10$ ) of compressibility-driven flames as a function of the heat loss parameter $b$ for small values of $\Lambda$; computed with $L e=1$ and $q=5$. The curves marked with open circles are shown in the figure insets on a refined scale. 


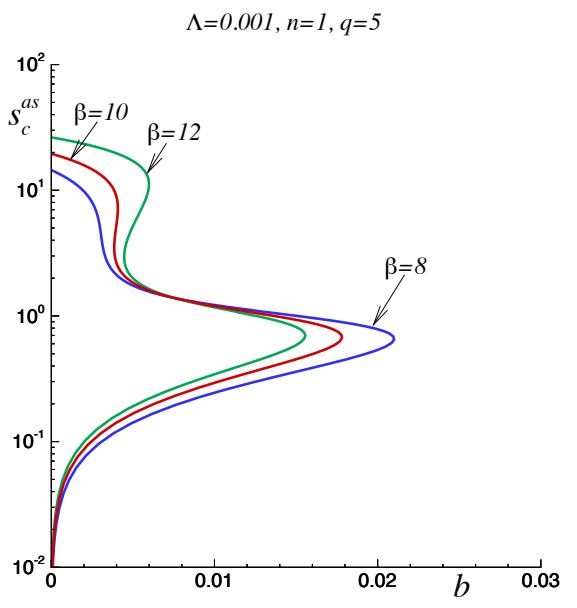

(a) $n=1$

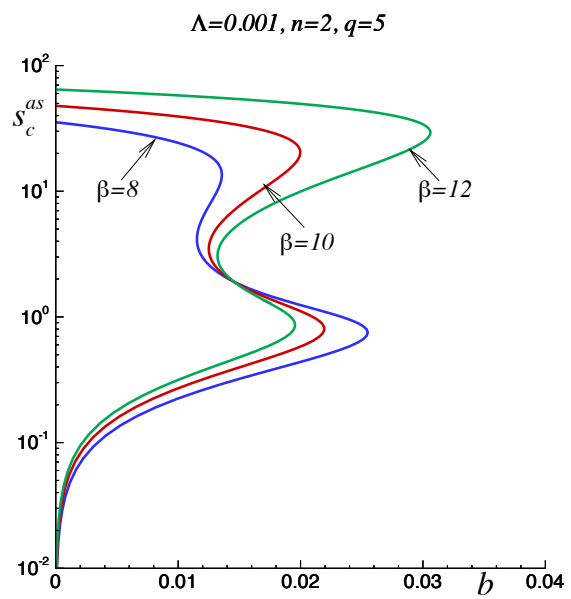

(b) $n=2$

Figure 11: The sensitivity of the propagation speed $s_{c}^{\text {as }}$ on the activation energy parameter $\beta$, for $\Lambda=0.001$ and the two exponents $n=1$ and $n=2$.

the segments with positive slope, where $s_{c}$ increases with increasing $b$, correspond to unstable states, the results can be interpreted as follows. When increasing the heat loss parameter $b$, starting from a nearly-adiabatic state, the flame speed decreases to a point where, instead of being extinguished (as in the case of a C-shaped curve) the flame propagates at a substantially slower speed that continues to decrease when further increasing $b$. In some cases, as for $\Lambda=0.01$ with $n=2$, the flame speed drops to a value corresponding to an isobaric flame. In all cases the flame is extinguished when $b$ exceeds the critical value $b_{c}$.

Finally, we note that, although the flame temperature $\theta_{f}$ and pressure $P_{f}$ of compressibilitydriven flames are, to leading order, independent of the activation energy parameter, the propagation speed depends significantly on $\beta$ as shown in Figure 11; see also the approximation given by Eq. (34). The (stable) isobaric flames, on the other hand, are practically independent of $\beta$.

\section{Conclusions}

In this study, we have investigated the structure and dynamics of compressibility-driven flames that evolve in long tubes closed at their ignition end, in the presence of heat losses through the tube walls. A narrow gap approximation is used to reduce the governing equations to an ef- 
fectively one-dimensional problem, with the reduced Mach number $\Lambda$ playing a key role on the flame behavior. In long channels this one-dimensional problem admits traveling-wave solutions that we have investigated numerically for finite values of the Zel'dovich number $\beta$, and asymptotically in the limit of $\beta \gg 1$. The structure of compressibility-driven flames, with $\Lambda>0$, is significantly different that the classical isobaric flames corresponding to $\Lambda=0$. The fresh unburned gas is first compressed and heated before it expands by the heat conducted from the reaction zone, and the pressure rises substantially throughout the entire wave. Under adiabatic conditions, the temperature of the burned gas trapped behind the flame attains the isochoric flame temperature, $T_{b}=T_{u}+Q Y_{u} / c_{v}$, which is much larger than the isobaric flame temperature $T_{a}=T_{u}+Q Y_{u} / c_{p}$, and the pressure attains the value $p_{b}=p_{u}\left(1+Q Y_{u} / c_{v} T_{u}\right)$. The propagation speed $\sim \exp \left(-E / 2 \mathcal{R} T_{b}\right)$ is much higher than the laminar flame speed $\sim \exp \left(-E / 2 \mathcal{R} T_{a}\right)$, and for the typical $\beta$ of combustible mixtures can be twenty to fifty times larger. A notable result is the weak dependence of the propagation speed on the compressibility parameter, which persists to very small values of $\Lambda$.

In the presence of heat losses we show that, similar to isobaric flames, the response curve that traces the dependence of the propagation speed on the heat loss parameter is C-shaped. Anticipating that the lower branch corresponds to unstable states, the solution branch along the upper branch corresponds to a flame that slows down when heat losses intensify and extinguishes (at the turning point) when heat losses become excessive. However, unlike isobaric flames, the fast compressibility-driven flames can better resist heat-losses, and survive under substantially larger values of the heat loss parameter $b$. The propagation speed at extinction is nearly half the speed of the adiabatic flames, and remains substantially high exceeding the laminar flame speed by ten-to-twenty times.

The results of the asymptotic analysis are in agreement with the numerical results for finite values of $\Lambda$. Moreover, the asymptotic approximation was able to capture and describe the transition from fast compressibility-driven to slow isobaric flames when systematically reducing the compressibility parameter $\Lambda$, a limit that is not easily accessible in numerical integrations. We show that when the characteristic Mach number is reduced and compressibility effects weaken, the response curve of the propagation speed vs heat loss has a more complex shape that exhibits a bistable behavior. For a given set of parameters (Mach number and heat loss), compressibilitydriven states corresponding to fast and slow flames coexist. Thus, a fast propagating flame will 


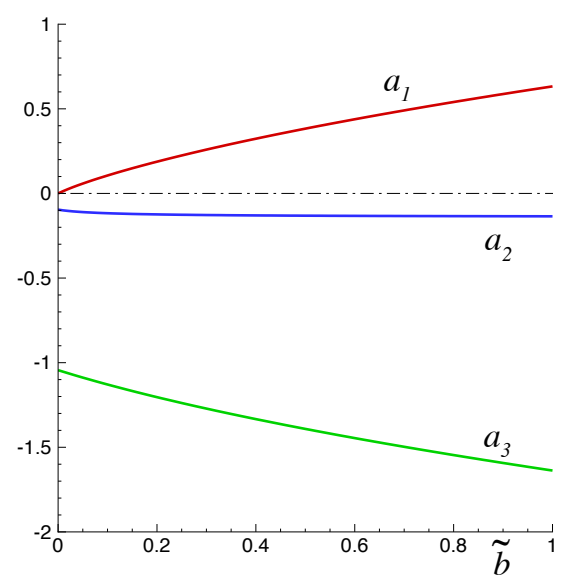

Figure 12: The roots of the cubic (A.46), for $\gamma=1.4$ and $\Lambda=0.1$, as a function of $\tilde{b}$.

abruptly transition to a slower flame when heat losses exceed a critical threshold; the slow flame continues to slow down when the heat losses intensify before extinguishing. At very low values of $\Lambda$ the compressibility-driven flame is highly sensitive to heat losses and could abruptly shift to an isobaric flame with a very small amount of heat loss.

\section{Acknowledgments}

V.N.K. acknowledges the support of Spanish MEC under project \#ENE2015-65852-C2-2-R (MINECO / FEDER, EU).

\section{Appendix A}

In this appendix, we describe the shooting method used to numerically solve the outer problem (38)-(39), subject to the jump conditions (40). The equations are solved in the finite domain $\zeta_{\min }<\zeta<\zeta_{\max }$, with $\zeta_{\min }$ and $\zeta_{\max }$ taken sufficiently large to ensure that the solution remains domain independent. Since $P \sim 0$ as $\zeta \rightarrow \pm \infty$, the asymptotic behavior of the solution for large $|\zeta|$ can be obtained from the linearized form of the equations. Eliminating $P$, yields a third order linear equation for $\theta$ with constant coefficients. The solution then takes the form

$$
\theta \sim \sum_{i=1}^{3} c_{i}^{ \pm} \mathrm{e}^{a_{i} \zeta}, \quad P \sim \sum_{i=1}^{3} \frac{\Lambda q}{a_{i}+\gamma \Lambda} c_{i}^{ \pm} \mathrm{e}^{a_{i} \zeta},
$$


where $c_{i}^{ \pm}$are arbitrary constants (the ${ }^{ \pm}$corresponding to positive/negative $\zeta$ ), and $a_{i}$ are the roots of the cubic

$$
a^{3}+(1+\Lambda \gamma) a^{2}+(\Lambda-\tilde{b}) a-\tilde{b} \Lambda \gamma=0 .
$$

It can be easily verified that for $\tilde{b}>0$ the three roots of (A.46) are real; one of them positive (say, $a_{1}>0$ ) and the other two negative $\left(a_{2}, a_{3}<0\right)$. The three roots for $\gamma=1.4$ and $\Lambda=0.1$, for example, are shown in Fig. 12 as a function of $\tilde{b}$. The implication is that

$$
\theta \sim c_{1}^{-} \mathrm{e}^{a_{1} \zeta}, \quad P \sim \frac{\Lambda q}{a_{1}+\gamma \Lambda} c_{1}^{-} \mathrm{e}^{a_{1} \zeta} \quad \text { as } \zeta \rightarrow-\infty,
$$

since $c_{2}^{-}=c_{3}^{-} \equiv 0$. The coefficient $c_{1}^{-}$can therefore be used as a shooting parameter. Starting with the asymptotic behavior (A.47), the equations are integrated from at $\zeta_{\min }$ to $\zeta=0$, where the jump conditions are applied, and then from $\zeta=0$ to $\zeta_{\max }$. The coefficient $c_{1}^{-}$is adjusted iteratively until the condition $c_{1}^{+}=0$ as $\zeta \rightarrow+\infty$ is satisfied, which ensures that $\theta \sim 0$ and $P \sim 0$ in the limit.

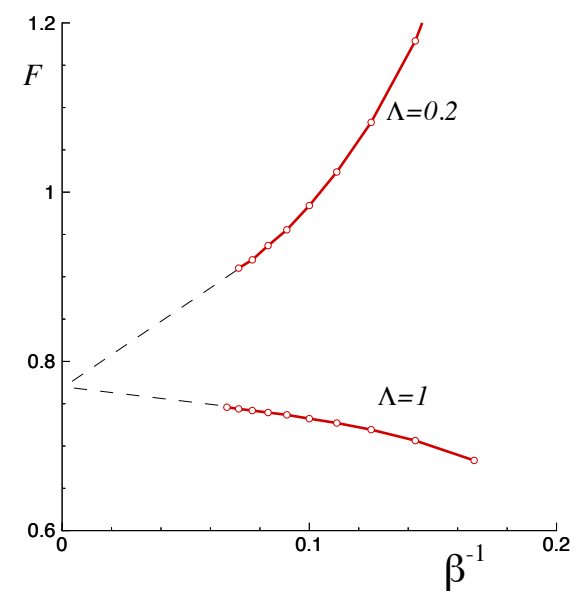

Figure 13: The dependence of $F$, defined in (B.49) on the expansion parameter $\beta^{-1}$ for two values of $\Lambda$ with $n=2$; the solid curve corresponds to the asymptotic approximation, the $\circ$ symbol to the numerically computed values, and the dashed curves to the linear extrapolation to $\beta^{-1}=0$. 


\section{Appendix B}

In this appendix, we discuss the nature of the asymptotic approximation of the propagation speed for large $\tilde{\beta}$ or equivalently for $\beta \gg 1$, focusing on the adiabatic case $(b=0)$. Extending the expansion of $s_{c}$ to higher orders, requires expanding the inner and outer expansions in power series of $\beta^{-1}$. With

$$
\begin{aligned}
& \theta_{f}=\gamma+\beta^{-1} \theta_{f_{1}}+\beta^{-2} \theta_{f_{2}}+\ldots, \\
& P_{f}=q+\beta^{-1} P_{f_{1}}+\beta^{-2} P_{f_{2}}+\ldots,
\end{aligned}
$$

equation (34) for the propagation speed takes the form

$$
s_{c}^{\mathrm{as}}=\underbrace{\frac{(1+q \gamma)^{2}}{(1+q)^{2-n / 2}} \exp \left\{\frac{\beta}{2} \frac{\gamma-1}{(1+q \gamma) /(1+q)}\right\}}_{E}\left(1+\frac{a_{1}}{\beta}+\frac{a_{2}}{\beta^{2}}+\frac{a_{3}}{\beta^{3}}+\ldots\right)
$$

where $a_{1}$ and $a_{2}$ are $O(1)$ constants, independent on $\beta$. We note parenthetically that (B.48) is not a classical asymptotic expansion, with well defined gauge functions, but has the form of a "composite expansion" consisting of the product of exponential and algebraic factors with different dependencies on $\beta$. The calculation of $a_{1}, a_{2}$, etc., involves a formidable task that will not attempted here. Rather we will validate the expansion (B.48) using the "exact" numerical solution of $s_{c}$. Plotted in Fig. 13 is the dependence of

$$
F=\beta\left(\frac{s_{c}}{E}-1\right) \sim a_{1}+\frac{a_{2}}{\beta}+\frac{a_{3}}{\beta^{2}}+\ldots
$$

on $\beta^{-1}$ for $\Lambda=1,0.2$ and $n=2$ with the dashed lines corresponding to the linear extrapolation to $\beta^{-1}=0$. The coefficients in the asymptotic expansion (B.48) can now be evaluated aposteriori:

$$
a_{1}=\lim _{\beta^{-1} \rightarrow 0} F, \quad a_{2}=\lim _{\beta^{-1} \rightarrow 0}\left[\beta\left(F-a_{1}\right)\right], \quad \text { etc. }
$$

The common value $a_{1} \approx 0.77$ (also read from Fig. 13), and the values of $a_{2}$ for $\Lambda=1$ and $\Lambda=0.2$ are $a_{2} \approx-0.33$ and $a_{2} \approx 1.98$, respectively. To this order $s_{c}^{\text {as }}$ is found to approach the "exact" numerical values of $s_{c}$ with an accuracy of less than $0.1 \%$ over the entire range of $\beta$ considered.

\section{References}

[1] L. Kagan, P. Gordon, G.I. Sivashinsky, An asymptotic study of the transition from slow to fast burning in narrow channels, Proc. Combust. Inst., 35 (2015) 913-920. 
[2] V.N. Kurdyumov, M. Matalon, Effects of gas compressibility on the dynamics of premixed flames in long narrow adiabatic channels, Combust. Theory \& Modelling 20 (2016) 1046-1067.

[3] V.N. Kurdyumov, M.Matalon, Flame acceleration in long narrow open channels, Proc. Comb, Ints. 34 (2013) 865872.

[4] G. Joulin and P. Clavin, Analyse asymptotiques des conditions d'extinction des flammes laminaires, Acta Astronautica, 3 (1976) 223-240.

[5] J. Buckmaster, The quenching of deflagation waves, Combust. Flame, 26 (1976) pp. 151-162.

[6] V.N. Kurdyumov, Propagation of premixed isobaric flames in narrow channels with heat-losses: The asymptotic analysis revised and reliance on the flame-sheet model, Combust. Flame, 206 (2019) 138-149.

[7] A. Liñán, The asymptotic structure of counterflow diffusion flames for large activation energies, Acta Asronautica, 1 (1974) 1007-1039. 